\title{
Agrin-Signaling Is Necessary for the Integration of Newly Generated Neurons in the Adult Olfactory Bulb
}

\author{
Katja Burk, ${ }^{1,2 \star}$ Angelique Desoeuvre, ${ }^{1,2 \star}$ Camille Boutin, ${ }^{1,2}$ Martin A. Smith, ${ }^{3}$ Stephan Kröger, ${ }^{4}$ Andreas Bosio, ${ }^{5}$ \\ Marie-Catherine Tiveron, ${ }^{1,2 \dagger}$ and Harold Cremer ${ }^{1,2 \dagger}$ \\ ${ }^{1}$ Aix-Marseille University, IBDML, 13288, Marseille, France, ${ }^{2}$ CNRS, UMR7288, 13288 Marseille, France, ${ }^{3}$ Department of Anatomy and Neurobiology, \\ University of California at Irvine, Irvine, California 92697, ${ }^{4}$ Ludwig-Maximilians-Universität München, 80336 Munich, Germany, and ${ }^{5}$ Miltenyi Biotec \\ GmbH, 51429 Bergisch Gladbach, Germany
}

In the adult forebrain, new interneurons are continuously generated and integrated into the existing circuitry of the olfactory bulb (OB). In an attempt to identify signals that regulate this synaptic integration process, we found strong expression of agrin in adult generated neuronal precursors that arrive in the olfactory bulb after their generation in the subventricular zone. While the agrin receptor components MuSK and Lrp4 were below detection level in neuron populations that represent synaptic targets for the new interneurons, the alternative receptor $\alpha 3-\mathrm{Na}^{+} \mathrm{K}^{+}$-ATPase was strongly expressed in mitral cells. Using a transplantation approach, we demonstrate that agrin-deficient interneuron precursors migrate correctly into the OB. However, in contrast to wild-type neurons, which form synapses and survive for prolonged periods, mutant neurons do not mature and are rapidly eliminated. Using in vivo brain electroporation of the olfactory system, we show that the transmembrane form of agrin alone is sufficient to mediate integration and demonstrate that excess transmembrane agrin increases the number of dendritic spines. Last, we provide in vivo evidence that an interaction between agrin and $\alpha 3-\mathrm{Na}^{+} \mathrm{K}^{+}$-ATPase is of functional importance in this system.

\section{Introduction}

In the adult mammalian forebrain, new neurons are generated throughout life by stem cell populations localized in the periventricular region. After their amplification, these cells undertake long distance migration into the olfactory bulb $(\mathrm{OB})$, where they differentiate into various types of interneurons using GABA, dopamine, or glutamate as their neurotransmitters (Lledo et al., 2008). Intense investigations of adult neurogenesis over the past decades led to the description of adult neural stem cells, their stepwise transformation into neurons, and the sequence of their synaptic integration (Lledo et al., 2008).

Currently, little is known about the molecular signals that regulate the synaptic integration of neurons into existing neuronal networks. In an attempt to identify such signals, we isolated migratory neuronal precursors from the subventricular zone (SVZ) and the rostral migratory stream (RMS) and analyzed their gene expression by Serial Analysis of Gene Expression (SAGE)

\footnotetext{
Received Sept. 27, 2011; revised Dec. 19, 2011; accepted Jan. 24, 2012.

Author contributions: K.B., A.D., M.-C.T., and H.C. designed research; K.B., A.D., and M.-C.T. performed research; M.A.S., S.K., and A.B. contributed unpublished reagents/analytic tools; K.B., A.D., C.B., A.B., M.-C.T., and H.C. analyzed data; M.-C.T. and H.C. wrote the paper.

This work has been supported by grants from the "Association Française contre le Myopathies," Agence National pour la Recherche (ForDopa), and the European Union (NoE Neurone, Axregen) to H.C.; and NIH Grant NS33213 to M.A.S. We thank Nathalie Coré, Antoine de Chevigny, and Christophe Beclin for critical reading of the manuscript, and Marion Gaudin for technical help. We thank Pico Caroni for providing Agrin-deficient mice.

*K.B. and A.D. are co-first authors.

${ }^{\dagger}$ M.-C.T. and H.C. are c0-senior authors.

Correspondence should be addressed to Harold Cremer, IBDML, Campus de Luminy case 907, 13288 Marseille cedex 9, France. E-mail: Harold.cremer@univmed.fr.

DOI:10.1523/JNEUROSCI.4906-11.2012

Copyright $\odot 2012$ the authors $\quad 0270-6474 / 12 / 323759-06 \$ 15.00 / 0$
}

(Pennartz et al., 2004) and microarray analysis (Boutin et al., 2010). One of the genes that showed strong and dynamic expression in this cell population was agrin.

Agrin, a proteoglycan existing in a variety of alternative splice forms, represents probably the best characterized synapseinducing factor and has been particularly investigated at the neuromuscular junction (Song and Balice-Gordon, 2008; Williams et al., 2008). Here, agrin and its receptor complex, comprising the low-density lipoprotein receptor Lrp4 and the muscle-specific kinase (MuSK), are essential for the formation and stabilization of this particular synapse (Gautam et al., 1996; Kim et al., 2008; Song and Balice-Gordon, 2008; Zhang et al., 2008). Agrin is widely expressed in the developing and adult brain where it has been shown to interact with the LRP4/MuSK complex (Ksiazek et al., 2007) as well as with the alternative receptor $\alpha 3-\mathrm{Na}^{+} \mathrm{K}^{+}$. ATPase ( $\alpha 3$ NKA) (Hilgenberg et al., 2006), suggesting that it might also play a role in neuron-to-neuron synapse formation (Hoch et al., 1993; O'Connor et al., 1994). Such a function is also supported by considerable amounts of in vitro data implicating agrin in processes like filopodia extension and spine formation (Ferreira, 1999; Maletic-Savatic et al., 1999; Bose et al., 2000; Annies et al., 2006; McCroskery et al., 2006; Matsumoto-Miyai et al., 2009; Ramseger et al., 2009). In vivo, analysis of agrin function in brain synapse formation has been difficult, due to the perinatal lethality of constitutive mouse mutants (Gautam et al., 1996; Lin et al., 2001). However, a lower amount of excitatory synapses in the postnatal cortex has been observed in agrin-deficient mice that were transgenically rescued (Ksiazek et al., 2007).

Here, we investigate the function of the agrin signaling pathway in adult neurogenesis. We demonstrate that agrin is necessary for 
morphological differentiation and survival of new $\mathrm{OB}$ interneurons and show that the transmembrane form of agrin (TM-agrin) is responsible for this function. Gain-offunction of TM-agrin induced excess synapses of new neurons in the OB. Finally, we provide evidence that $\alpha 3 \mathrm{NKA}$ is an active agrin receptor in this system.

\section{Materials and Methods}

Mice. Animals were treated according to guideline approved by the French Ethical Committee. Agrin mutants (Lin et al., 2001) (provided by P. Caroni, Friedrich Miescher Institute, Basel, Switzerland) were crossed with actin-EGFP transgenic mice (Hadjantonakis et al., 1998; Lin et al., 2001) in our animal facilities to generate donor mice. C57BL6 females (Charles River) aged from 7 to 10 weeks were used as host in the transplantation experiment.

RNA probes and in situ hybridization. cDNA fragments from mouse Lrp4 (772 bp; position 6323-7095), mouse MuSK (909bp; position 2210-3119), mouse $\alpha 3$ NKA (Atp1a3; 744bp; position 3112-3856), and mouse agrin (320bp; 5160-5479) were cloned in pGEMT vector (Promega) and used as described (Tiveron et al., 2006).

Transplantations. E18.5 embryos were analyzed for GFP fluorescence; agrin expression status was determined by PCR [primers for the wild-type (WT) allele: forward 5'-CAGGGGATAGTTGAGAAG-3', reverse 5' -GCTGGGATCT CATTGGTC- ${ }^{\prime}$; for the mutant allele: forward $5^{\prime}$-TCGCAAGTTCTAATTC CA-3', reverse 5'-GGGCAGGGCTAACACCAA-3']. SVZ tissue from the lateral wall of the anterior lateral ventricle was transplanted in host animals (1.5 $\mathrm{mm}$ posterior to bregma, $0.8 \mathrm{~mm}$ lateral, and $2.4 \mathrm{~mm}$ deep) as described by Seidenfaden et al. (2006).

Matrigel assay. Cultures and analyses of SVZ explants were performed as previously described (Hack et al., 2002).

Postnatal electroporation. (Boutin et al., 2008). Mouse agrin-specific siRNA (siRNA3) and mock siRNA vectors were previously described (McCroskery et al., 2006). Both vectors were coelectroporated with a pCAGGS_EGFP vector for detection and morphological analyses. A chicken $6 \mathrm{~kb}$ TM-agrin fragment was cloned in the appropriate orientation (TM-agrin) or in the reverse orientation (TM-agrinREV) into the bicistronic expression vector pCAGGS_IRES_EGFP, which allows doubtless identification of transfected cells. This fragment encodes the active form of agrin, the $y 4 z 8$ variant that additionally contains 4 aa at the $y$ site and 8 aa at the $z$ site (O'Connor et al., 1994; Neumann et al., 2001). For electroporation, mock siRNA was coelectroporated with TMagrinREV to serve as control, and siRNA3 was coelectroporated with either TM-agrinREV or TM-agrin. C-Ag15 was fused to the murine Ig $\kappa$ chain leader sequence and cloned into pCAGGS_IRES_EGFP.

Quantification and statistical analysis. Categorization and spine count were performed blind to experimental condition. Data are presented as mean \pm SEM. The designation $n$ represents the number of animals analyzed, except in Figures $3 d$ and $4 c$, where it represents the number of dendrites counted in 6-12 animals. Student's unpaired $t$ test was used to assess differences between data groups using Instat software (GraphPad Software). Differences were considered statistically significant when $p<0.05$.

\section{Results}

In a SAGE-based study (Pennartz et al., 2004) we found a strong over-representation of agrin transcripts in purified adult olfactory interneuron precursors (Pennartz et al., 2004) in comparison to total brain tissue. In agreement, in situ hybridization and immunohistochemistry showed strong signals in the RMS (Fig. 1a) as well as in the granule cell layer (GCL), the mitral cell layer (MCL), and the glomerular layer (GL) (compare Fig. 1a, arrow, http://mouse.brain-map.org/gene/show/11390).

We investigated the expression components of the well described agrin signaling pathway that is functional at the neuromuscular synapse (Song and Balice-Gordon, 2008). In the $\mathrm{OB}$, expression of MuSK was below detection level (compare Fig. 1c, http://mouse.brain-map.org/gene/show/17965), while Lrp4 was restricted to the GL but was undetectable in the MCL, containing the main target population for newly arriving interneurons (compare Fig. 1d, http://mouse.brain-map. org/gene/show/86700). In contrast, mRNA for the alternative agrin receptor in the CNS, $\alpha 3$ NKA (Hilgenberg et al., 2006), was strongly expressed in the MCL, more weakly in the GCL and GL, and was absent from the RMS (compare Fig. 1b, http://mouse.brain-map.org/gene.show/87535).

Thus, $\alpha 3$ NKA was the only known agrin receptor for which we could detect an expression in mitral cells, the cell population that represents the main synaptic targets for new interneurons.

Next, we investigated the function of agrin in adult olfactory neurogenesis. Given that agrin-deficient mice die perinatally (Lin et al., 2001), precluding the analysis of postnatal events like olfactory bulb synaptogenesis, we used a transplantation approach. Mice deficient for all agrin isoforms (Lin et al., 2001) were crossed with an actin-GFP transgenic line to allow recognition of graftderived neurons. (Hadjantonakis et al., 1998). SVZ tissue from mutant embryos or littermate controls at the latest possible time point, E18.5, was transplanted into the SVZ of adult WT host mice (Fig. 2a) (Seidenfaden et al., 2006). Ten days postgrafting (dpg), both control and agrin-deficient transplanted cells were found in the host RMS (Fig. $2 b$ ). In the center of the OB, transplanted cells separated from the main stream, switching from tangential chain migration to individual radial migration (Fig. $2 b$ ). No difference was obvious between genotypes. We used Matrigel culture of SVZ explants to confirm these observations in a more controlled and quantifiable system (Hack et al., 2002). Here, WT and agrin-deficient explants showed the same typical migratory behavior, with cells exiting from the core-aggregate in 
a

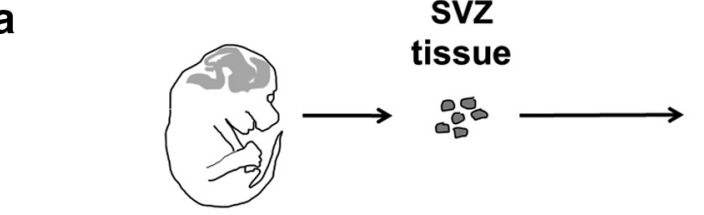

E18 donor: Agrin wt; actin-GFP or Agrin ${ }^{--}$; actin-GFP

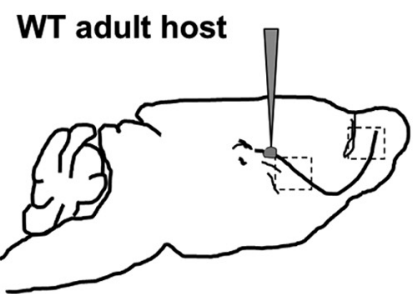

d

C
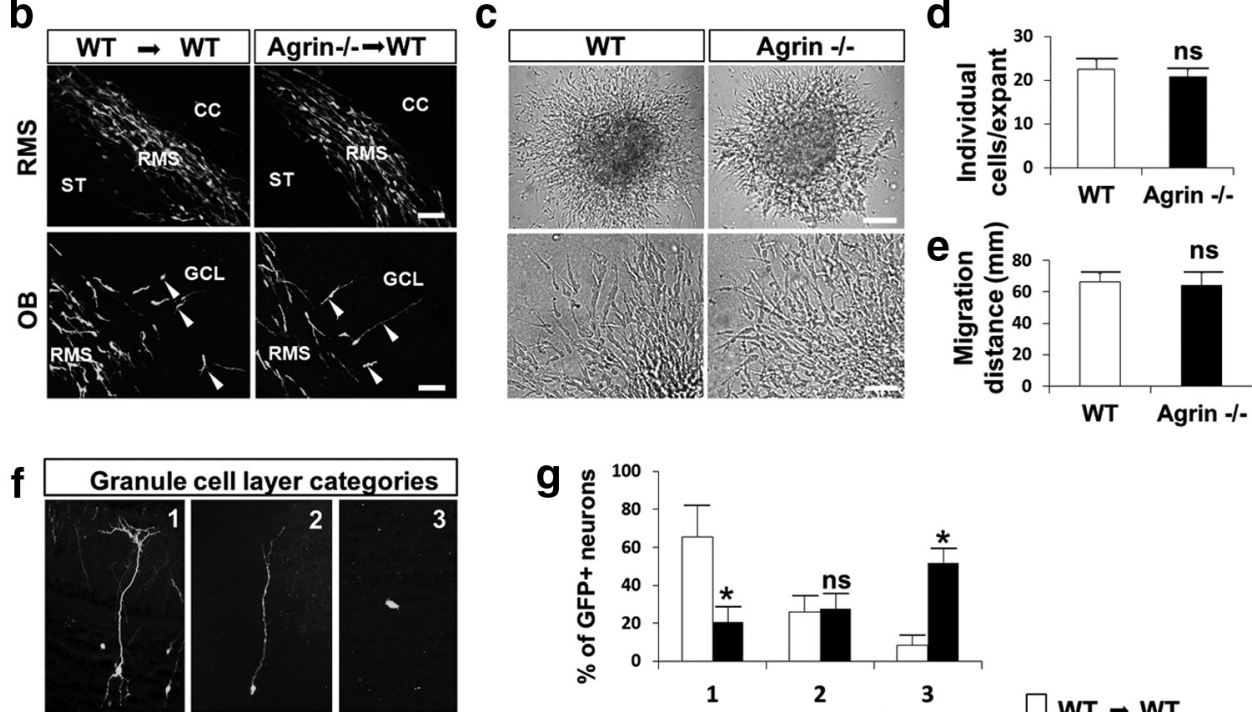

9

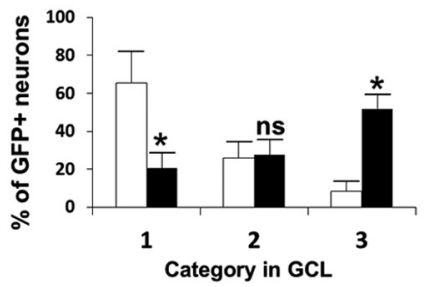

$\square$ WT $\rightarrow$ WT

h Glomerular layer categories

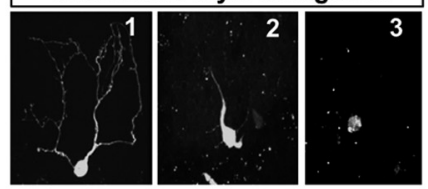

i

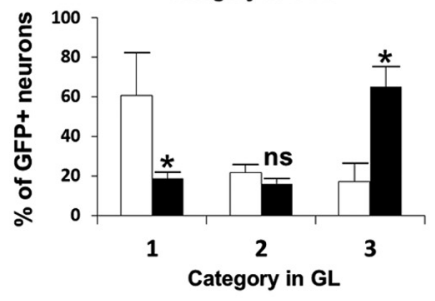

Agrin $-/-W T$
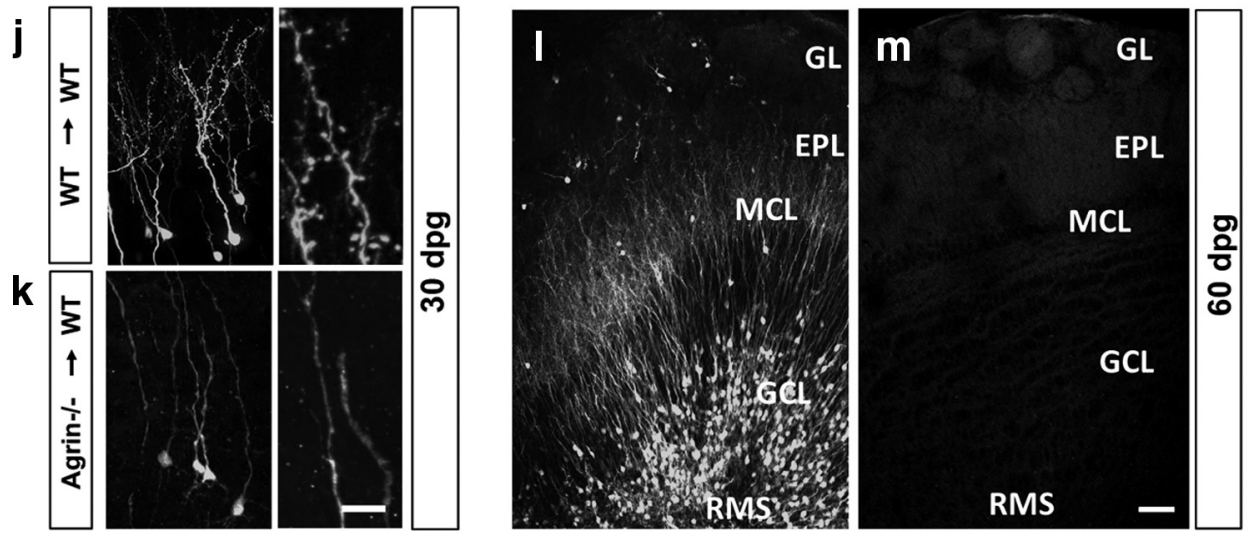

Figure 2. Agrin-deficient neuronal precursors do not integrate in the OB. $\boldsymbol{a}$, Schematic representation of the experimental protocol. GFP-labeled WT or agrin-deficient precursors isolated at E18.5 were transplanted into the anterior SVZ of adult WT hosts. $\boldsymbol{b}$, In the RMS, organization and distribution of mutant precursors were indistinguishable from controls (top panels). In the center of the OB, WT and agrin-deficient precursors showed the typical switch from tangential chain to radial individual migration (bottom panels, arrowheads). c, Matrigel culture of WT and agrin-deficient explants isolated at E18.5. Bottom panels are high-magnification images of the explants presented in the top panels. $\boldsymbol{d}, \boldsymbol{e}$, In both situations, explants showed the typical chain migration with no differences in the number of individual cells surrounding the explant $(\boldsymbol{d})$ or migration distance $(\boldsymbol{e}) . \boldsymbol{f}, \boldsymbol{h}$, Examples for morphological categories of neurons in the GCL $(\boldsymbol{f})$ and in the GL $(\boldsymbol{h})$ as used for quantification. $\boldsymbol{g}, \boldsymbol{i}$, At $17 \mathrm{dpg}$, cells with mature morphologies (category 1) were significantly reduced when agrin-deficient tissue was transplanted $\left(n=6\right.$; control: $n=5$; $\left.{ }^{*} p<0.05\right)$, while simple morphologies (category 3) were over-represented in both the GCL $(\boldsymbol{g})$ and the $\mathrm{GL}(\boldsymbol{i}) . \boldsymbol{j}, \boldsymbol{k}$, At $30 \mathrm{dpg}$, integrated WT granule cells show mature morphology and dense coverage in the external plexiform layer (EPL) with dentritic spines (j). Right, High-magnification images of dendrites presented in left panels. At this time point, surviving agrin-deficient cells are rare. The few remaining cells show few signs of branching or protrusions $(\boldsymbol{k}) . \boldsymbol{I}, \boldsymbol{m}, \mathrm{At} 60 \mathrm{dpg}$, animals transplanted with WT SVZ tissue show generally large amounts of fully mature neurons in the $0 B(\boldsymbol{I})$. At this time point, mice grafted with mutant tissue were always devoid of GFP-positive cells $(\boldsymbol{m})$. Scale bars: $\boldsymbol{b}, 20 \mu \mathrm{m} ; \boldsymbol{c}$, top panels, $100 \mu \mathrm{m} ; \boldsymbol{c}$, bottom panels, $40 \mu \mathrm{m} ; \boldsymbol{j}, \boldsymbol{k}$, left panels, $15 \mu \mathrm{m} ; \boldsymbol{j}, \boldsymbol{k}$, right panels, $5 \mu \mathrm{m}$; $I, m, 100 \mu \mathrm{m}$. CC, corpus callosum; ST, striatum; ns, non-significant. 


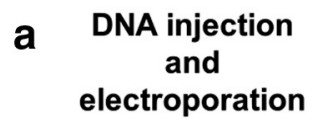
aNA injection electroporation

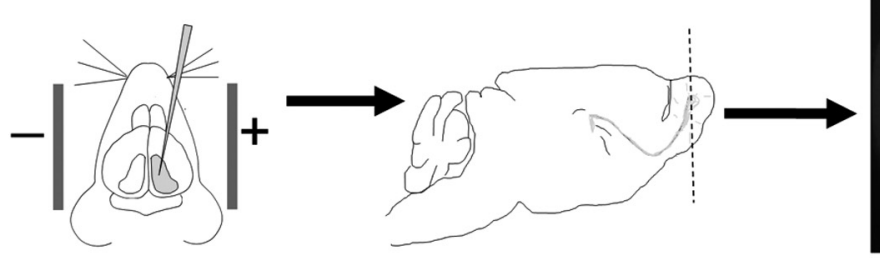

\section{Olfactory Bulb \\ Coronal section}

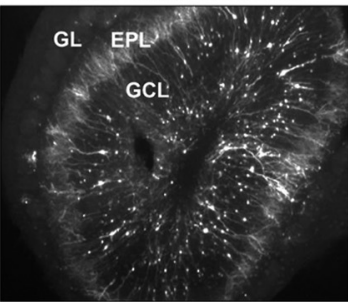

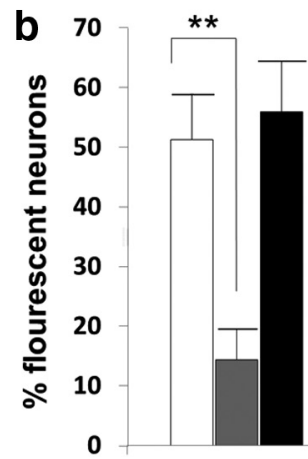

1

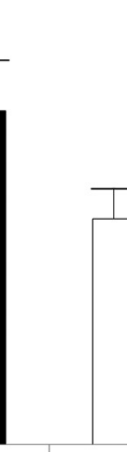

2

\section{Category in GCL}

C

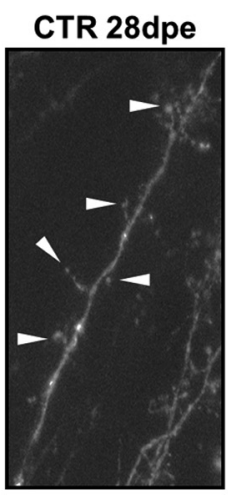

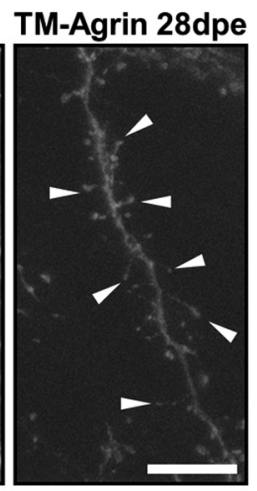

d

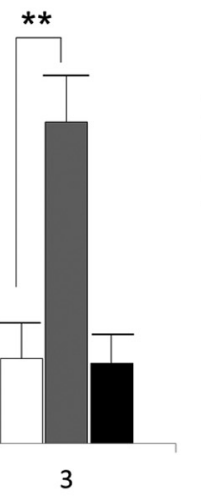

$\square$ Control

siRNA

siRNA + TM-Agrin
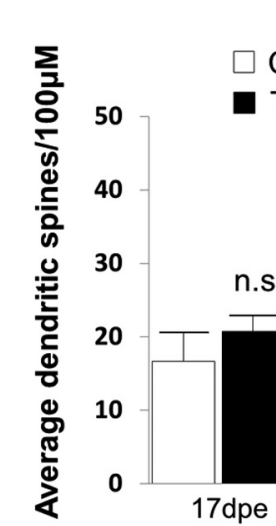

Control

TM-Agrin

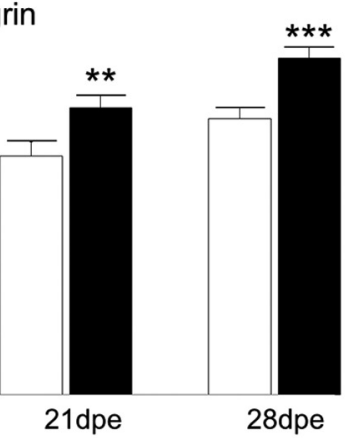

Figure 3. TM agrin is the active agrin isoform. $\boldsymbol{a}$, Representation of the electroporation protocol. $\boldsymbol{b}$, Morphological distribution of the electroporated cells in the $\mathrm{GCL}$ at $21 \mathrm{dpe}$. siRNA-induced knockdown of TM-agrin leads to the loss of cells with mature neuronal morphology in the GCL (category 1), while simple cells (category 3 ) are over-represented. This effect is entirely rescued by coelectroporation of a TM-agrin expression construct ( $n=6$ for control; $n=10$ for siRNA; $n=10$ for TM-agrin rescue). $\boldsymbol{c}, \boldsymbol{d}$, Gain-of-function of TM-agrin leads to an increasing number of dendritic spines in the EPL (17 dpe, $n=20$ and 32 dendrites for control and TM; $21 \mathrm{dpe}, n=26$ and 55 dendrites for control and TM; $28 \mathrm{dpe}, n=91$ and 100 for control and TM. ${ }^{* *} p<0.01$, ${ }^{* * *} p<0.001$, n.s. non significant, Student's $t$ test). Scale bar, $30 \mu \mathrm{m}$.

a chain-like organization (Fig. 2c). The numbers of individual cells surrounding the explants (Fig. $2 d$ ) or migration distance (Fig. 2e) were indistinguishable. We conclude that the migratory behavior of agrin mutant neuronal precursors was normal.

Next, we investigated the fate of RMS-transplanted neuronal precursors after their arrival in the OB. For quantification of the differentiation state, we defined the following three categories of cellular morphologies in the GCL (Fig. $2 f$ ) and GL (Fig. $2 h$ ): first, multibranched cells with the morphology of mature neurons (category 1); second, cells with one main process and little or no secondary branching (category 2); and third, cells showing no signs of process outgrowth or branching (category 3 ).

Ten days postgrafting, the category distribution of cells in the $\mathrm{OB}$ after grafting of control or mutant tissue was comparable (data not shown). However, at $17 \mathrm{dpg}$ both GCL and GL of hosts transplanted with mutant grafts contained a significantly lower percentage of complex cell types of category 1 , while cells that showed no signs of neuronal morphology (category 3) were strongly over-represented (Fig. $2 g, i$ ). At $30 \mathrm{dpg}$, transplantation of WT precursors led to integration of large numbers of cells with mature neuronal morphology and covered with dendritic spines in the $\mathrm{OB}$ (Fig. 2j). At this time point, agrin-deficient transplanted cells were rare. The few surviving cells that were observed in the GCL showed simple morphologies and lacked dendritic spines (Fig. $2 k$ ). At $60 \mathrm{dpg}$, hosts transplanted with WT tissue showed generally large amounts of fully mature neurons integrated in the $\mathrm{OB}$ (Fig. $2 l$ ), while animals with mutant grafts were always devoid of GFP-positive cells (Fig. $2 m$ ). Thus, agrin deficiency compromised integration and survival of new interneurons in the OB.

Alternative splicing leads to the generation of secreted and membrane-anchored forms of agrin (Burgess et al., 2000; Neumann et al., 2001). The TM form is the main form expressed in 


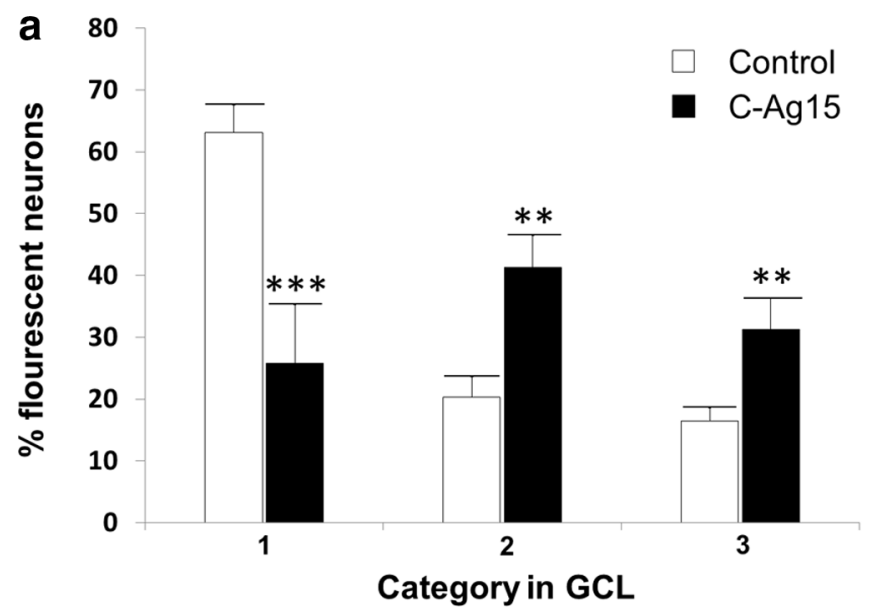

b CTR C15
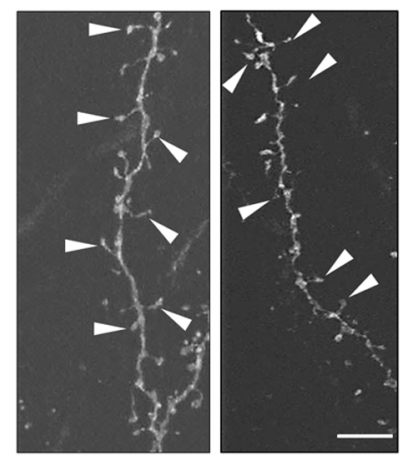

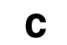

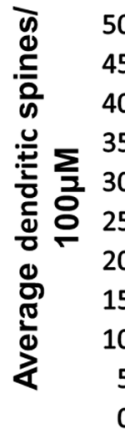

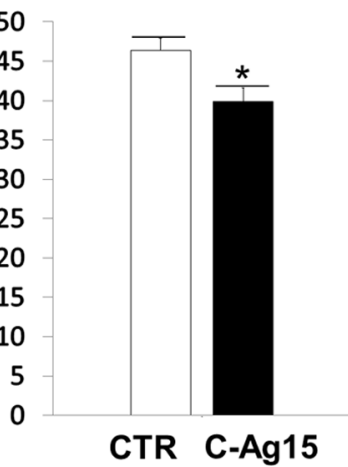

Figure 4. Expression of the competitive agrin antagonist C-Ag15 interferes with differentiation and spine formation in the $\mathrm{OB}$. $\boldsymbol{a}$, Electroporation of a C-Ag15 expression construct leads to the loss of cells with mature neuronal morphology and the overrepresentation of category 2 and 3 cells in the $\mathrm{GCL}\left(n=8\right.$ for $\mathrm{C}-15 \mathrm{Ag}$ and for control; ${ }^{* *} p<0.01,{ }^{* * *} p<0.001$, Student's $t$ test). $\boldsymbol{b}, \boldsymbol{c}$, In parallel to the shift in categories, spine density is significantly reduced in the category 1 cells that show extensions into the EPL (control: $n=69$, C-Ag15: $n=59 ;{ }^{*} p<0.05$, Student's $t$ test). Scale bar, $10 \mu \mathrm{m}$.

the CNS and has been implicated in process formation and synaptogenesis (Annies et al., 2006; McCroskery et al., 2006). We asked whether transmembrane agrin was the functional isoform in the OB. Postnatal forebrain electroporation allows the efficient manipulation of new neurons that invade the OB via the RMS (Fig. 3a) (Boutin et al., 2010). Transfection of a knock-down vector expressing a well characterized siRNA that specifically targeted agrin mRNA (McCroskery et al., 2006), together with EGFP for detection, induced the expected over-representation of cells with simple morphologies at the expense of mature phenotypes at $21 \mathrm{~d}$ postelectroporation (dpe) (Fig. $3 b$ ). This effect was entirely rescued by coelectroporation of the anti-agrin siRNA together with a TM-agrin expression vector (Fig. $3 b$ ). Next, we investigated the consequences of TM-agrin gain-of-function. Electroporation of the TM-agrin expression vector in wild-type mice had no influence on the morphological maturation of new neurons at $21 \mathrm{dpe}$ (data not shown). However, average spine density in the external plexiform layer (EPL) was significantly increased at this time point and even higher at $28 \mathrm{dpe}$ (Fig. $3 c, d$ ). Altogether, these data demonstrate that the TM form of agrin is sufficient for the correct morphological differentiation of new $\mathrm{OB}$ interneurons and suggest that this effect is due to formation and/or stabilization of their synapses.

The above expression data suggest that $\alpha 3$ NKA rather than MuSK/Lrp4 is involved in mediating agrin signaling in the OB. We aimed at providing functional evidence for possible agrin- $\alpha 3$ NKA interactions. A C-terminal fragment of agrin, C-Ag15, has been shown to competitively antagonize interaction between endogenous agrin and the $\alpha 3 \mathrm{NKA}$ (Hilgenberg et al., 2006; Tidow et al., 2010). We expressed C-Ag15, fused to the murine Ig $\kappa$ chain leader sequence for secretion, together with EGFP using postnatal brain electroporation and categorized the morphology of new interneurons at $21 \mathrm{dpe}$. These analyses revealed that expression of C-Ag15 induced a loss of mature phenotypes in the GCL and an increase of category 3 cell types, comparable to the results obtained by siRNA-mediated knockdown (compare Figs. $4 a, 3 b$ ) or the transplantation studies (Fig. 2g). Furthermore, the small number of type 1 granule neurons that extended branched processes into the EPL showed a significant decrease in dendritic spine number compared with controls (Fig. $4 b, c)$. These results are in agreement with an antagonistic function of C-Ag15 in agrin signaling and suggest that $\alpha 3 \mathrm{NKA}$ is a functional receptor.

\section{Discussion}

Unlike neurons that are born in the embryo, adult neuronal precursors of the $\mathrm{OB}$ have to induce and stabilize synapses in a mature and fully functional circuitry. The mechanisms underlying this integration are beginning to be elucidated. For example, it is now evident that neuronal activity is a key factor in the process regulating both survival and synaptic integration (Kelsch et al., 2010). In contrast, nothing is known about the actual molecular cross talk that mediates the formation and stabilization of new synapses in the adult brain. Our work strongly suggests that agrin is an essential mediator of this interaction. Agrin is strongly expressed in neuronal precursors in the RMS and $\mathrm{OB}$ (O'Connor et al., 1994; Cohen et al., 1997). We find that agrin-deficient neurons transplanted into a wild-type forebrain show normal migration in the RMS and OB. However, at later stages mutant neurons show few dendritic spines and are eliminated, pointing to a function of the proteoglycan in synaptic integration. This is further supported by our analysis of the TM isoform of agrin, which we find to be necessary for morphological differentiation and sufficient for the induction of additional dendritic spines in the EPL.

In our expression studies, we cannot detect MuSK expression in the OB and find that LRP4 is present in the periglomerular layer and in proximal regions of the RMS, but not in the major target population for new neurons in the OB, the mitral cells. Although these findings are in agreement with data in the Allen Brain Atlas (http://mouse.brain-map.org/welcome.do), expression of Lrp4 in different layers of the OB, including mitral cells, has been found by others (Tian et al., 2006). We are left with this discrepancy, which awaits clarification.

However, we find that the ion-pump $\alpha 3$ NKA, which has been identified as an alternative agrin receptor (Hilgenberg et al., 2006), is particularly strongly expressed in the OB. It has been shown that an interaction between agrin and the competitive 
agrin inhibitory fragment C-Ag15 produces an agrin mutant-like phenotype. Thus, the ion transporter might be the functional receptor in this system.

Interestingly, another brain region in which agrin is particularly strongly expressed and in which $\alpha 3$ NKA reaches comparably high levels as in the $\mathrm{OB}$ is the hippocampal formation, which also shows adult neurogenesis and therefore permanent synapse formation. It appears conceivable that, like in the OB, the controlled integration of new neurons in hippocampal neurogenesis depends on agrin signaling.

So far, the only in vivo study that approached agrin function in the CNS was based on agrin-deficient mice in which perinatal lethality was rescued by transgenic expression of the proteoglycan in motorneurons (Ksiazek et al., 2007). These animals survive to adulthood and show largely normal brain and neuron morphology except for an $\sim 30 \%$ reduction in the number of presynaptic and postsynaptic excitatory specialization. The fact that a functional and largely normal brain can develop in the absence of agrin is, at least at first glance, at odds with our finding of a total inability of new interneurons to maintain synapses and survive in the OB. However, in our transplantation and RNAi experiments agrin-deficient precursors have to compete for synaptic integration with coincidently arriving cells that express normal amounts of agrin, while in the transgenic rescue situation all cortical neurons permanently lack the proteoglycan. This indicates that agrin is not essential for synapse formation in the CNS, but that it provides merely a selective advantage. Interestingly, our expression data suggest that in the OB MuSK/Lrp4, which mediates neuromuscular junction formation in an all-or-nothing fashion, might not be the main functional receptor complex. Instead, the alternative receptor $\alpha 3 \mathrm{NKA}$ is strongly expressed by the cell populations that represent synaptic targets for new neurons in the $\mathrm{OB}$. Inhibition of this ion transporter by agrin induces a reduction in membrane potential and increases neuron excitability (Hilgenberg et al., 2006). In this scenario, agrin-deficient neurons would be selectively disadvantaged, in agreement with their loss after transplantation in WT hosts, further supporting the notion that neuronal activity is a key factor for the integration of neurons in the OB.

\section{References}

Annies M, Bittcher G, Ramseger R, Löschinger J, Wöll S, Porten E, Abraham C, Rüegg MA, Kröger S (2006) Clustering transmembrane-agrin induces filopodia-like processes on axons and dendrites. Mol Cell Neurosci 31:515-524.

Bose CM, Qiu D, Bergamaschi A, Gravante B, Bossi M, Villa A, Rupp F, Malgaroli A (2000) Agrin controls synaptic differentiation in hippocampal neurons. J Neurosci 20:9086-9095.

Boutin C, Diestel S, Desoeuvre A, Tiveron MC, Cremer H (2008) Efficient in vivo electroporation of the postnatal rodent forebrain. PLoS One 3:e1883.

Boutin C, Hardt O, de Chevigny A, Coré N, Goebbels S, Seidenfaden R, Bosio A, Cremer H (2010) NeuroD1 induces terminal neuronal differentiation in olfactory neurogenesis. Proc Natl Acad Sci U S A 107:1201-1206.

Burgess RW, Skarnes WC, Sanes JR (2000) Agrin isoforms with distinct amino termini: differential expression, localization, and function. J Cell Biol 151:41-52.

Cohen NA, Kaufmann WE, Worley PF, Rupp F (1997) Expression of agrin in the developing and adult rat brain. Neuroscience 76:581-596.

Ferreira A (1999) Abnormal synapse formation in agrin-depleted hippocampal neurons. J Cell Sci 112:4729-4738.

Gautam M, Noakes PG, Moscoso L, Rupp F, Scheller RH, Merlie JP, Sanes JR (1996) Defective neuromuscular synaptogenesis in agrin-deficient mutant mice. Cell 85:525-535.

Hack I, Bancila M, Loulier K, Carroll P, Cremer H (2002) Reelin is a detachment signal in tangential chain-migration during postnatal neurogenesis. Nat Neurosci 5:939-945.
Hadjantonakis AK, Gertsenstein M, Ikawa M, Okabe M, Nagy A (1998) Generating green fluorescent mice by germline transmission of green fluorescent ES cells. Mech Dev 76:79-90.

Hilgenberg LG, Su H, Gu H, O'Dowd DK, Smith MA (2006) Alpha3Na+/ $\mathrm{K}+-\mathrm{ATP}$ ase is a neuronal receptor for agrin. Cell 125:359-369.

Hoch W, Ferns M, Campanelli JT, Hall ZW, Scheller RH (1993) Developmental regulation of highly active alternatively spliced forms of agrin. Neuron 11:479-490.

Kelsch W, Sim S, Lois C (2010) Watching synaptogenesis in the adult brain. Annu Rev Neurosci 33:131-149.

Kim N, Stiegler AL, Cameron TO, Hallock PT, Gomez AM, Huang JH, Hubbard SR, Dustin ML, Burden SJ (2008) Lrp4 is a receptor for agrin and forms a complex with MuSK. Cell 135:334-342.

Ksiazek I, Burkhardt C, Lin S, Seddik R, Maj M, Bezakova G, Jucker M, Arber S, Caroni P, Sanes JR, Bettler B, Ruegg MA (2007) Synapse loss in cortex of agrin-deficient mice after genetic rescue of perinatal death. J Neurosci 27:7183-7195.

Lin W, Burgess RW, Dominguez B, Pfaff SL, Sanes JR, Lee KF (2001) Distinct roles of nerve and muscle in postsynaptic differentiation of the neuromuscular synapse. Nature 410:1057-1064.

Lledo PM, Merkle FT, Alvarez-Buylla A (2008) Origin and function of olfactory bulb interneuron diversity. Trends Neurosci 31:392-400.

Maletic-Savatic M, Malinow R, Svoboda K (1999) Rapid dendritic morphogenesis in CA1 hippocampal dendrites induced by synaptic activity. Science 283:1923-1927.

Matsumoto-Miyai K, Sokolowska E, Zurlinden A, Gee CE, Lüscher D, Hettwer S, Wölfel J, Ladner AP, Ster J, Gerber U, Rülicke T, Kunz B, Sonderegger P (2009) Coincident pre- and postsynaptic activation induces dendritic filopodia via neurotrypsin-dependent agrin cleavage. Cell 136:1161-1171.

McCroskery S, Chaudhry A, Lin L, Daniels MP (2006) Transmembrane agrin regulates filopodia in rat hippocampal neurons in culture. Mol Cell Neurosci 33:15-28.

Neumann FR, Bittcher G, Annies M, Schumacher B, Kröger S, Ruegg MA (2001) An alternative amino-terminus expressed in the central nervous system converts agrin to a type II transmembrane protein. Mol Cell Neurosci 17:208-225.

O'Connor LT, Lauterborn JC, Gall CM, Smith MA (1994) Localization and alternative splicing of agrin mRNA in adult rat brain: transcripts encoding isoforms that aggregate acetylcholine receptors are not restricted to cholinergic regions. J Neurosci 14:1141-1152.

Pennartz S, Belvindrah R, Tomiuk S, Zimmer C, Hofmann K, Conradt M, Bosio A, Cremer H (2004) Purification of neuronal precursors from the adult mouse brain: comprehensive gene expression analysis provides new insights into the control of cell migration, differentiation, and homeostasis. Mol Cell Neurosci 25:692-706.

Ramseger R, White R, Kröger S (2009) Transmembrane form agrininduced process formation requires lipid rafts and the activation of Fyn and MAPK. J Biol Chem 284:7697-7705.

Seidenfaden R, Desoeuvre A, Bosio A, Virard I, Cremer H (2006) Glial conversion of SVZ-derived committed neuronal precursors after ectopic grafting into the adult brain. Mol Cell Neurosci 32:187-198.

Song Y, Balice-Gordon R (2008) New dogs in the dogma: Lrp4 and Tid1 in neuromuscular synapse formation. Neuron 60:526-528.

Tian QB, Suzuki T, Yamauchi T, Sakagami H, Yoshimura Y, Miyazawa S, Nakayama K, Saitoh F, Zhang JP, Lu Y, Kondo H, Endo S (2006) Interaction of LDL receptor-related protein 4 (LRP4) with postsynaptic scaffold proteins via its C-terminal PDZ domain-binding motif, and its regulation by $\mathrm{Ca} /$ calmodulin-dependent protein kinase II. Eur J Neurosci 23:2864-2876.

Tidow H, Aperia A, Nissen P (2010) How are ion pumps and agrin signaling integrated? Trends Biochem Sci 35:653-659.

Tiveron MC, Rossel M, Moepps B, Zhang YL, Seidenfaden R, Favor J, König $\mathrm{N}$, Cremer H (2006) Molecular interaction between projection neuron precursors and invading interneurons via stromal-derived factor 1 (CXCL12)/CXCR4 signaling in the cortical subventricular zone/intermediate zone. J Neurosci 26:13273-13278.

Williams S, Ryan C, Jacobson C (2008) Agrin and neuregulin, expanding roles and implications for therapeutics. Biotechnol Adv 26:187-201.

Zhang B, Luo S, Wang Q, Suzuki T, Xiong WC, Mei L (2008) LRP4 serves as a coreceptor of agrin. Neuron 60:285-297. 